\title{
A Signal Processing Approach for the Failure Analysis of Rolling-Element Bearing of Vehicle Brake Tester Used at a Vehicle Inspection Station
}

\author{
Selman Kulaç \\ Department of Electrical-Electronics Engineering \\ Faculty of Engineering, Duzce University \\ Duzce, TURKEY \\ selmankulac@duzce.edu.tr
}

\begin{abstract}
In this study, failure analysis of rolling-element bearing of vehicle brake tester used in a vehicle inspection station was considered according to the real time vibration signals. Based on vibration measurements under loads taken at intervals of about 15 days, these real time vibration signals were subjected to median filtering firstly. The total difference method proposed with this paper using autocorrelation functions' maximum and minimum values for each vibration signal was applied. Lastly, Goertzel algorithm has been used in order to obtain amplitude of specific defect frequency sample with a minimum calculation complexity for vibrational failure analysis of rolling-element bearing for the first time with this paper. It is verified with this paper that as the time passes, the values obtained by all methods above increase and excess the thresholds determined by this paper.

Index Terms-Failure analysis, rolling-element bearing, vibration measurement, vibration signal processing, vehicle roller brake tester, signal processing
\end{abstract}

\section{INTRODUCTION}

Vehicle braking systems are controlled periodically for their decisive importance in vehicle safety. The control of vehicle brakes are determined by the vehicle roller braking testers at vehicle inspection stations. Drum cylinders used in vehicle brake testers are mounted on rolling element bearings. Rolling element bearing fault is one of the most common causes of vehicle brake tester malfunctions. Therefore, failure analysis of rolling-element bearings used in a vehicle brake testers is necessary.

A considerable amount of literature has been published on signal processing based vibration monitoring and interpretations in order to determine bearing life and faults. Failure detection and diagnosis for a class of rolling-element bearings using short time FFT (Fast Fourier Transform)-based methods were investigated by Yang et al. [1]. Special auto-correlation analysis in frequency domain with an aim to distinguish the faults in the bearings was accomplished by Ming et al. [2]. The effect of sensor noise is noticeable in that study. In [3], vibration analysis in monitoring rolling-element bearings was

978-1-7281-1003-5/19/\$31.00 @2019 IEEE summarized and their capabilities, advantages and disadvantages were explained. In [4], the comparable efficiency of using frequency domain approach in HilbertHuang Transform for bearing fault diagnosis was handled. But empirical mode decomposition process existing in this study causes mode mixing problem. In [5], as the time passes, it is stated that the sum of the frequency amplitude values above the certain thresholds in frequency domain increases for the rolling-element bearing of a vehicle brake tester in loaded and unloaded cases. In [6], explicit finite element modelling of a rolling element bearing with a localised line spall using standard signal processing techniques etc. is obtained and analytical validation of the modelled results is presented. In [7], a novel modulation signal bispectrum (MSB) based robust detector for bearing fault detection which is more accurate and robust detection results than Kurtogram based approaches is proposed.

Although rolling element bearings have been widely used on brake testers at vehicle inspection stations, the bearing problems have not been considered before. In this study, a signal processing approach for the failure analysis of rollingelement bearings used at a vehicle inspection station was considered according to the real time vibration signals.

Novelties of this study are presented below.

In experimental :

- Taking the real time vibration signals from vehicle inspection station was performed.

In theory:

- The total difference method proposed with this paper for the first time using autocorrelation functions' maximum and minimum values for each vibration signal was applied for vibrational fault diagnosis of rolling-element bearing.

- Goertzel algorithm has been used in order to obtain amplitude of specific defect frequency sample with a minimum calculation complexity for vibrational fault diagnosis of rolling-element bearing for the first time with this paper.

- It is verified with this paper that as the time passes, the values obtained by all methods (total difference 


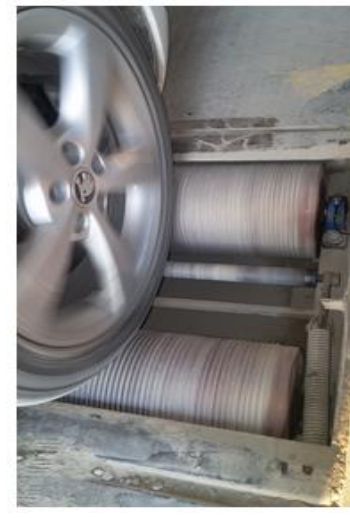

(a)

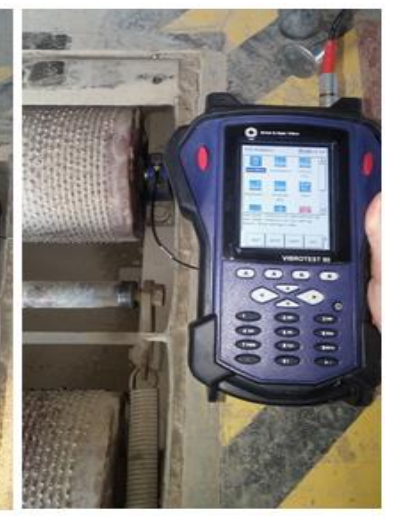

(b)

Fig. 1. Pictures from experimental setup [5]

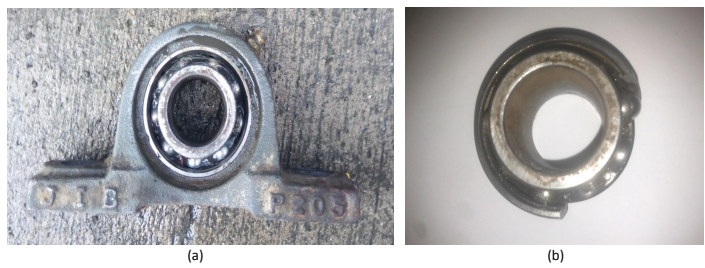

Fig. 2. Used bearing sample

based autocorrelation and Goertzel algorithm) increase and excess the thresholds determined by this paper.

\section{Real Time Vibration Signals Obtaining And} ANALYSIS

The picture of the experimental setup is shown in Fig. 1 (a) and data acquisition (DAQ) device having accelorometer mounted on the bearing housing is shown in Fig. 1 (b). The bearing type is UCP205 and samples of healthy and broken down bearings are shown in Fig. 2. Two rolling element bearings are used for comparison. The first one was used to take measurement 1,2 and 3 data and the other one was used to take measurement 4 and 5 data for comparison. Vibration measurements for bearings of vehicle roller brake testers were performed at a vehicle inspection station for intervals of about 15 days. Five measurements were carried out on experimental setup on different dates. The first bearing had been mounted on the brake test system at the vehicle inspection station before first measurement and was in standard active use. The first measurement was taken while active use was in progress. The measurements were then continued at regular intervals and the first bearing was broken about 35 days after the first measurement. It should be noted that the life of the first bearing is longer than one month.

Median filtering is a non-linear method for removing noise from images. It is widely used because it is very effective at removing noise and outliers while preserving edges in image processing. From Fig. 3 and Fig. 4, the expected peak values are observed approximately two in one second depending on the rotational speed of the bearing. The rotational speed of the bearing in $\mathrm{Hz}$ is calculated by Eq. (1). The median filter was used instead of the mean filter to reduce noise without touching these peak values which were considered as edges. All recorded vibration measurement data for each of three axes in time domain were filtered by Median Filter in order to remove noise and spikes.

$$
N_{H z}=\frac{5000}{2 * \pi * r * 60 * 60}=2.1897 \mathrm{~Hz}
$$

In the formula, $\mathrm{r}$ is the radius of the brake drum equals to $0.101 \mathrm{~m}$.

Amplitude values of all filtered vibration data were obtained by using all axes data for each time samples and it is reached to the amplitude vector as a 1-dimensional signal in time domain and transferred with FFT to frequency domain in MATLAB. The three-axis accelerometer produces simultaneous data on three axes (x, y and $\mathrm{z})$ in each time slot. When this data in each time slot is considered as vector, in order to perform onedimensional signal processing, the amplitude of the vector is taken as it requires only one value instead of three values in each time slot and calculated by Eq. (2). The amplitude or norm or length or magnitude of this vector is known as absolute value. Therefore, negative parts are not included because absolute value expressions cannot be negative. In addition, amplitudes in frequency domain are given to show which frequencies have peaks. As seen in the frequency domain representations from Fig. 3 and Fig. 4, increasing of the peak value in $213.4 \mathrm{~Hz}$ used by the Goertzel algorithm will also be verified in next section.

$$
A_{v_{i}}=\sqrt{m_{x_{i}}^{2}+m_{y_{i}}^{2}+m_{z_{i}}^{2}}
$$

These processes are repeated for each measurement. The collected vibration signals in time domain and frequency domain are shown in Fig. 3 and Fig. 4 with recording date. For all signals, total number of samples (N) is 8192 and sampling period (Ts) is 0.000390625 second.

\section{Proposed Approach ANd Evaluations}

The autocorrelation function is generated when one of the same copy of the signal is shifted relative to the original one and calculated by Eq. (3).

$$
R_{x}[n]=\sum_{m=-\infty}^{\infty} x[m+n] x[m]
$$

Autocorrelation functions for all measurements are presented in Fig. ??. According to these autocorrelation functions, the total difference method proposed with this paper was applied. According to this method, the difference between the summation of maximum peak values and minimum peak values were calculated by Eq. (4) and presented in Fig. 6.

$$
D=\left(\sum_{i=1}^{N} A_{i}^{\max }\right)-\left(\sum_{i=1}^{N} A_{i}^{\min }\right)
$$



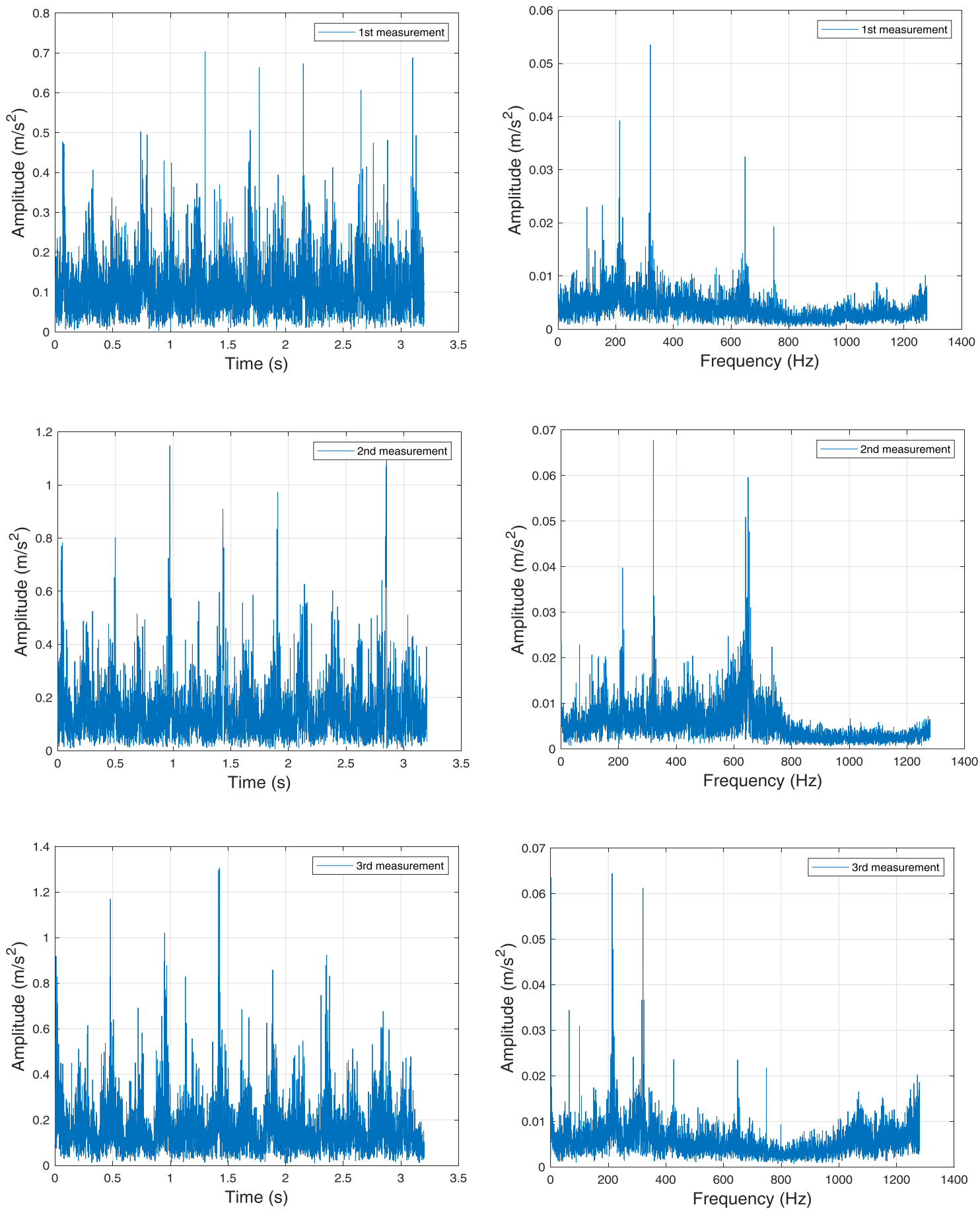

Fig. 3. Time and frequency domain representation of vibration amplitude signals of measurements of first rolling-element bearing

As can be understood from the Fig. 6, as the time passes, difference values of the vibration signals increase. The difference value before the first bearing was broken down, reached to peak value of 5967 with the 3rd measurement. This value can be regarded as the threshold level. After the first bearing was broken down, the difference between the summation of 

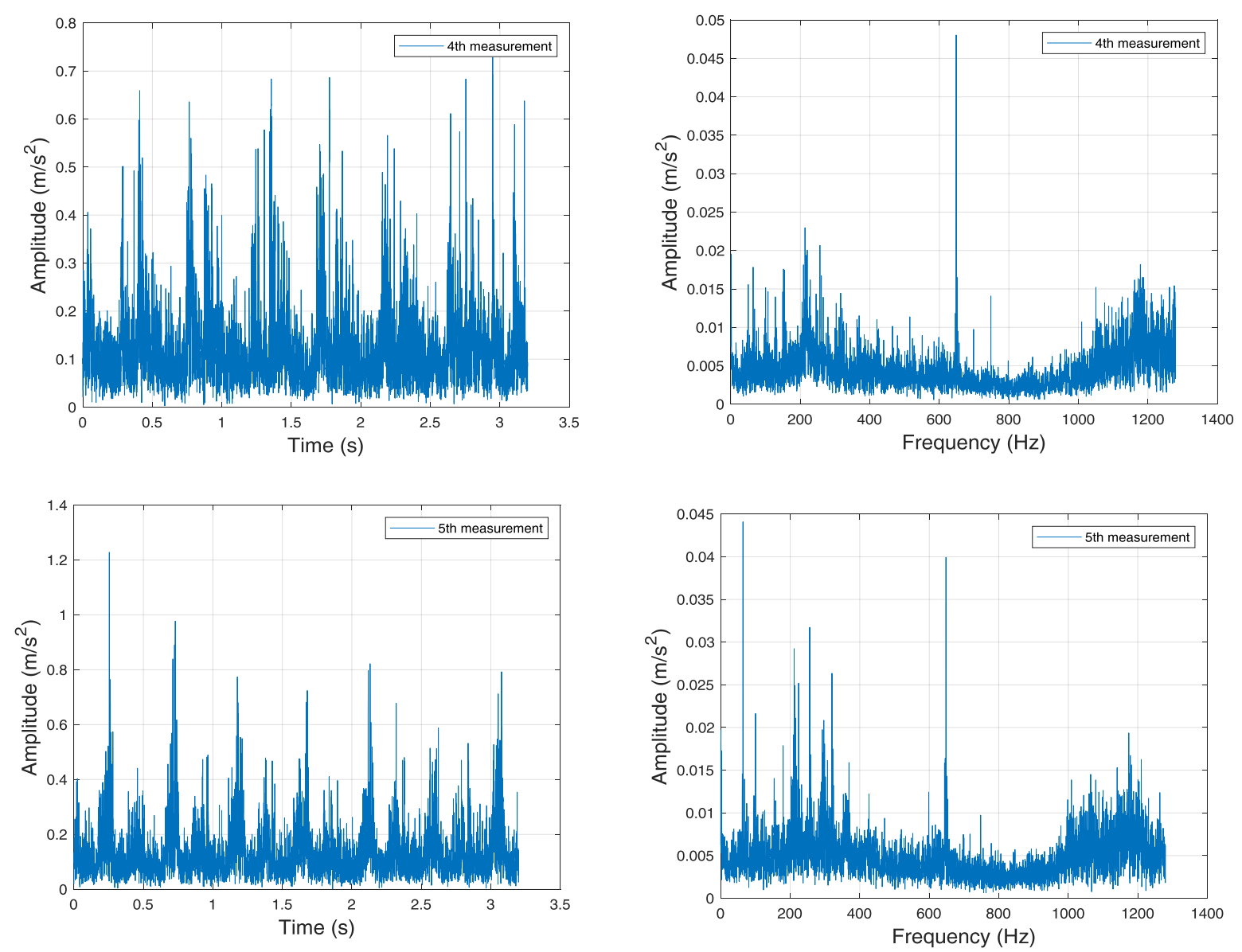

Fig. 4. Time and frequency domain representation of vibration amplitude signals of measurements of second rolling-element bearing

maximum peak values and minimum peak values for the second ball bearing increased from lower to higher value over time.

FFT algorithm is utilized in order to calculate DFT (Discrete Fourier Transform). When a few or specific DFT frequency samples are only needed, Goertzel algorithm can be used to decrease calculation complexity more whereas obtaining all the frequency samples [8]. The Goertzel algorithm is considerably superior to ensure energy efficiency against the FFT with less computational load, since less computational load causes less energy consumption [9].

Amplitude calculation of specific frequency sample with the Goertzel algorithm, which needs only signal energy without phase information, using the previous input values is calculated as

$$
\begin{array}{r}
X_{G}^{2}(k)=Q_{k}^{2}(N-1)+Q_{k}^{2}(N-2) \\
-2 \cos \left(2 \pi \frac{k}{N}\right) Q_{k}(N-1) Q_{k}(N-2)
\end{array}
$$

where $Q_{k}$ s are intermediate values that are formed when entering the input signals in time domain in the Goertzel algorithm.

In mechanical signal analysis literature for the rollingelement bearings, the Goertzel algorithm is utilized for the first time with this study. The Goertzel algorithm is used to obtain predetermined and specific spectral amplitude of 213.4 $\mathrm{Hz}$ frequency sample in this paper. $213.4 \mathrm{~Hz}$ frequency sample corresponds to outer raceway defect frequency harmonic and calculated by Eq. (6). As can be understood from the Fig. 7, as the time passes, spectral amplitudes of $213.4 \mathrm{~Hz}$ frequency samples of the vibration signals increase. The final measurement (3rd one) before the first bearing was broken down, spectral amplitude of $213.4 \mathrm{~Hz}$ frequency sample reached to peak value of $0.0644 \mathrm{~m} / \mathrm{s}^{2}$. This value can be regarded as the threshold level. After the first bearing was broken down, spectral amplitude of $213.4 \mathrm{~Hz}$ frequency sample for the second ball bearing increased from lower to higher value over time. 

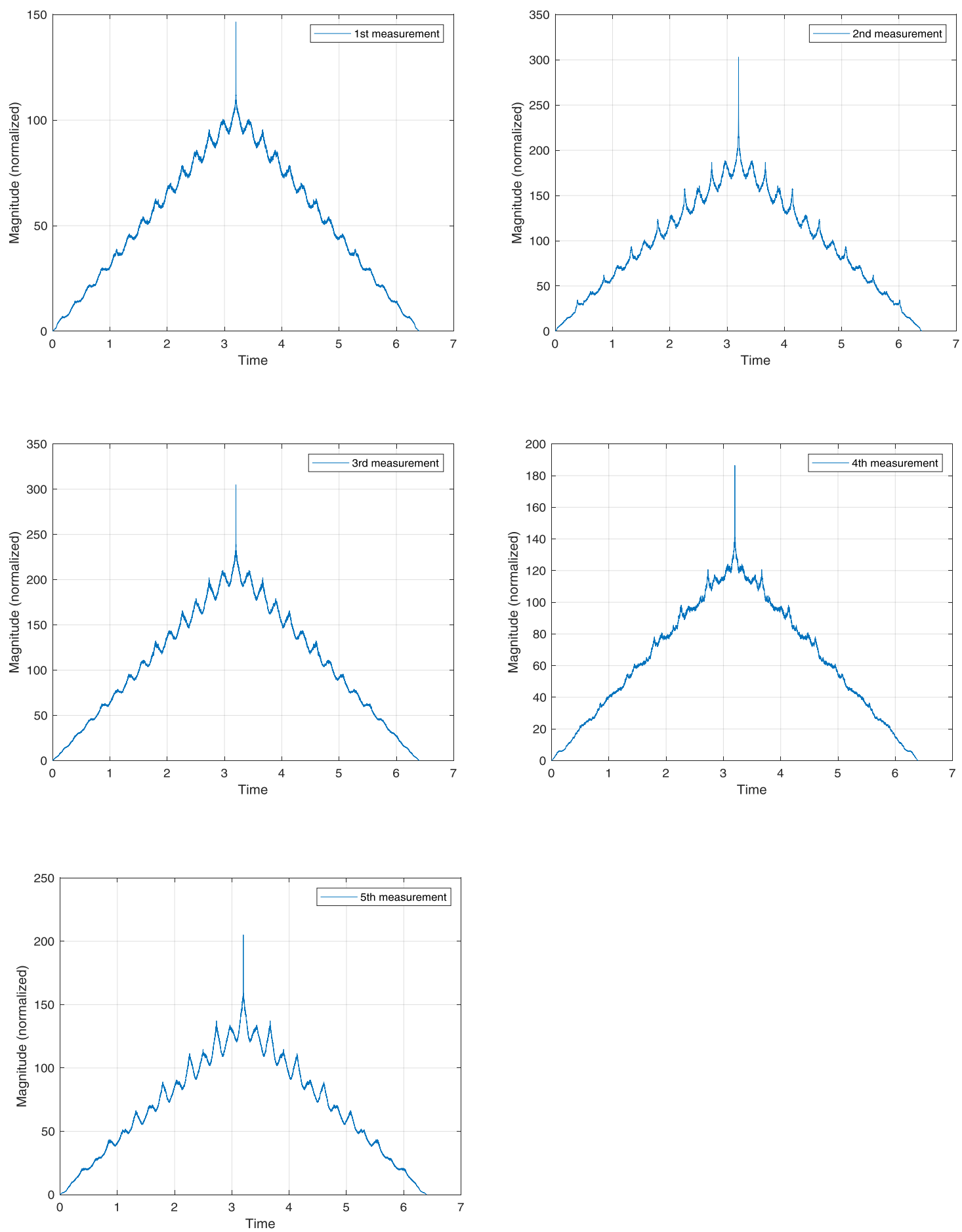

Fig. 5. Autocorrelation functions for all measurements

$$
O R F=\left(\frac{n}{2}\right)\left(N_{H z}\right)\left(1-\frac{d}{D} \cos \alpha\right)
$$

Outer raceway defect frequency (ORF) is calculated as $8.0771 \mathrm{~Hz}$ according to the formula above where $N_{H z}$ is the RPM of the shaft in $\mathrm{Hz}$ equals to $2.1897 \mathrm{~Hz}$, d is the mean 


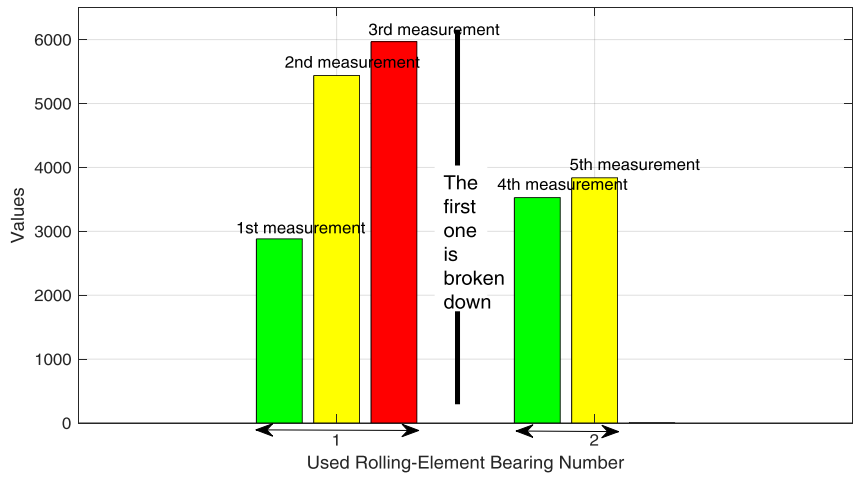

Fig. 6. The difference values according to the total difference method

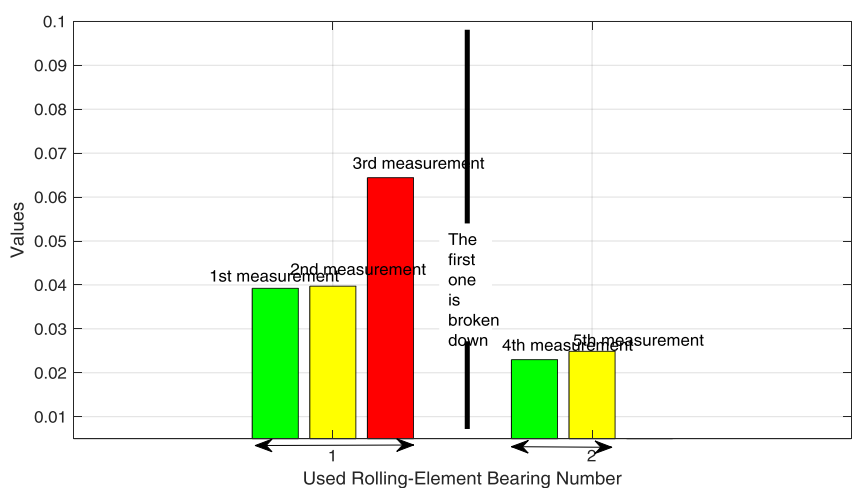

Fig. 7. Spectral amplitude values of $213.4 \mathrm{~Hz}$ frequency samples

diameter of the rolling elements equals to $7.40 \mathrm{~mm}$, D is the pitch diameter of the bearing equals to $41 \mathrm{~mm}, \mathrm{n}$ is the number of rolling elements equals to 9 and $\alpha$ is the contact angle equals to $0.213 .4 \mathrm{~Hz}$ is the 26th harmonic of ORF.

\section{CONCLUSION}

Safety and efficiency of vehicle roller brake tester used at vehicle inspection stations are important for reliable inspection. Rolling element bearing used in the brake tester play important role for effective vehicle inspection tests. In this study, pre-alarming and failure analysis of rollingelement bearing of vehicle brake tester used at a vehicle inspection station was considered by taking the real time vibration signals. Real time vibration signals were subjected to autocorrelation difference and Goertzel algorithm methods after median filtering process. At this point, it will be possible to obtain an effective prediction of rolling element bearing failures in the brake testers at vehicle inspection stations.

\section{ACKNOWLEDGMENT}

The author gratefully thanks Assoc. Prof. Dr. Suat Sardemir for helping during experiments.

\section{REFERENCES}

[1] YANG, Z., U.C. MERRILD, M.T. RUNGE, G. PEDERSEN, and H BRSTING. A study of rolling-element bearing fault diagnosis using motor's vibration and current signatures, IFAC Proceedings Volumes, vol. 42, no. 8, pp. 354- 359, 2009, 7th IFAC Symposium on Fault Detection, Supervision and Safety of Technical Processes. [Online]. Available: http://www.sciencedirect.com/science/article/pii/S1474667016358025

[2] MING, A., Z. QIN, W. ZHANG, and F. CHU. Spectrum autocorrelation analysis and its application to fault diagnosis of rolling element bearings, Mechanical Systems and Signal Processing, vol. 41, no. 1, pp. 141 - 154, 2013. [Online]. Available: http://www.sciencedirect.com/science/article/pii/S0888327013003798

[3] BOUDIAF, A., A. DJEBALA, H. BENDJMA, A. BALASKA and A. DAHANE. A summary of vibration analysis techniques for fault detection and diagnosis in bearing, in 2016 8th International Conference on Modelling, Identification and Control (ICMIC), Nov 2016, pp. 37-42.

[4] RAI, V.and A.MOHANTY, Bearing fault diagnosis using $\mathrm{fft}$ of intrinsic mode functions in hilberthuang transform, Mechanical Systems and Signal Processing, vol. 21, no. 6, pp. 2607 - 2615, 2007. [Online]. Available: http://www.sciencedirect.com/science/article/pii/S0888327006002846

[5] KULAC, S. and S. SARIDEMIR, Evaluation of vibration spectral values of arolling-element bearing used in a vehicle inspection station. 3rd International Conference on Engineering and Natural Science ICENS 2017, 2017.

[6] SINGH, S. C.Q. HOWARD, C.H. HANSEN AND U.G. KOPKE. Analytical validation of an explicit finite element model of a rolling element bearing with a localised line spall, Journal of Sound and Vibration, vol. 416, pp. 94 - 110, 2018. [Online]. Available: http://www.sciencedirect.com/science/article/pii/S0022460X17306703

[7] TIAN, X., J.X. GU, I.REHAB, G.M. ABDALLA, F.GU and A.BALL. A robust detector for rolling element bearing condition monitoring based on the modulation signal bispectrum and its performance evaluation against the kurtogram, Mechanical Systems and Signal Processing, vol. 100, pp. 167 - 187, 2018. [Online]. Available: http://www.sciencedirect.com/science/article/pii/S0888327017304004

[8] GOERTZEL, G. An algorithm for the evaluation of finite trigonometric series, The American Mathematical Monthly, vol. 65, no. 1, pp. pp. 3435, 1958. [Online]. Available: http://www.jstor.org/stable/2310304

[9] WANG, W., G.ZHIBIN, L.HUANG and Y.YAO. Spectrum sensing based on goertzel algorithm, pp. 1-4, 112008. 